\title{
Magnetic perturbations seen by CHAMP and evaluated using the TIE-GCM
}

\author{
D. T. Mozzoni ${ }^{1,2}$, M. Mandea ${ }^{1}$, and J. Cain ${ }^{3}$ \\ ${ }^{1}$ GeoForschungsZentrum Potsdam, Telegrafenberg, 14473 Potsdam, Germany \\ ${ }^{2}$ Florida State University, Department of Physics, Tallahassee, FL 32312, USA \\ ${ }^{3}$ Florida State University, Geophysical Fluid Dynamics Institute, Tallahassee, FL 32312, USA
}

Received: 8 March 2007 - Revised: 16 June 2007 - Accepted: 29 June 2007 - Published: 30 July 2007

\begin{abstract}
The Thermosphere-Ionosphere Electrodynamics General Circulation Model (TIE-GCM) is a self-consistent, global, atmospheric model that can be used to estimate magnetic perturbations at satellite altitude. These computed perturbations can then be compared with the magnetic vector data provided by low-earth orbiting satellites. In this initial study, the quietest day of each month from 2001-2005 was selected for comparison. CHAMP magnetic vector residuals were computed for these intervals using the CHAOS model to remove core and crustal geomagnetic contributions. Under various input parameters, the TIE-GCM predictions were compared with the CHAMP residuals on an orbit by orbit basis. Initial results demonstrate a reasonable agreement between the TIE-GCM estimates and the CHAMP residuals in non-polar, dayside regions $\left( \pm 50^{\circ}\right.$ magnetic latitude) where both are able to resolve the Equatorial Electro-Jet (EEJ) and solar quiet (Sq) current systems. Although no clear component or temporal correlation was discerned, evidence showing the decrease in residual comparisons presents the possibility of using the TIE-GCM to pre-process geomagnetic data for main field modeling purposes.
\end{abstract}

Keywords. Geomagnetism and paleomagnetism (General or miscellaneous) - Ionosphere (Mid-latitude ionosphere)

\section{Introduction}

When either a magnetic observatory or a satellite records a geomagnetic field measurement, it represents the superposition of many sources. The largest contribution arises from the approximately dipolar main (or core) field being generated by geodynamic processes within the fluid, iron-rich, outer core of the Earth. However, depending upon the altitude and location of the measurement, it may contain sizable contri-

Correspondence to: D. T. Mozzoni

(dmozzoni@gfz-potsdam.de) butions from the static lithospheric field, originating largely from rocks within the Earth's crust. Another significant portion comes from external field sources, which are produced in the ionosphere and magnetosphere. These variable sources include the daily solar quiet $(\mathrm{Sq})$ and ring current variations, the contributions from the Disturbance Storm Time $\left(D_{s t}\right)$ deviations, and the many other current systems (e.g., the fieldaligned currents and the auroral and equatorial electro-jets) present in the geospace region. Currents induced in the solid Earth by these external fields must also be considered. In satellite data it is even possible to resolve small signals attributable to the electrical currents generated by ocean flow (Tyler et al., 2003). Most of these field sources undergo temporal variations, both periodic and non-periodic, ranging from the secular variation of the main field on decadal scales down to the sub-second variations during geomagnetic storms in the magnetosphere.

Understanding and interpreting these magnetic field contributions and their origins is a major task; however, significant progress has recently been achieved using ground and satellite based data. The present study is an effort to better understand the external sources of the geomagnetic field by first evaluating the prospect of comparing a physicsbased model derived using limited direct observational input with that of actual, observed, geomagnetic data. One aim here is that this comparison may in turn help with the development of future geomagnetic main field models. For this we have used the Thermosphere-Ionosphere Electrodynamics General Circulation Model (TIE-GCM) (Richmond et al., 1992), developed at the National Center for Atmospheric Research (NCAR) in Boulder, Colorado. This model can be used to simulate many different atmospheric quantities, including wind velocities, various atmospheric species concentrations, temperatures, electric fields, and current densities. The current densities can later be post-processed to compute magnetic perturbations both above and below the ionosphere. In order to validate these model results, comparisons

Published by Copernicus Publications on behalf of the European Geosciences Union. 
of the predicted perturbations calculated at the altitude of the CHAMP satellite (taken to be $430 \mathrm{~km}$ ) with vector residuals computed from the difference in the CHAMP data and the CHAOS geomagnetic model (Olsen et al., 2006) were studied.

In the present study, the quietest day of each month from 2001-2005 according to the list of international Q-days, was selected. New residuals were then computed for these quiet days between the original CHAMP/CHAOS data residuals and estimates from the different TIE-GCM model runs. In this paper we show that the TIE-GCM model can, to some degree, reproduce the residuals computed from CHAMP geomagnetic vector data, and that it might be possible to use the TIE-GCM to pre-process dayside satellite data in order to supplement geomagnetic modeling efforts, especially when there is a scarcity of quality data.

\section{Data and models}

This study is based upon the combination of satellite data provided by the CHAMP mission and two different models, the TIE-GCM and CHAOS, all of which are briefly described in the remainder of the section.

\subsection{CHAMP spacecraft and data}

The Challenging Minisatellite Payload (CHAMP) is a lowearth orbiting German satellite ${ }^{1}$ (Reigber et al., 2002). Since its launch in 2000, this multi-mission satellite has supplied invaluable, high precision, magnetic, gravity, and ion drift measurements. The essentially circular, near-polar orbit (87.3 $3^{\circ}$ with respect to the equator) of CHAMP allows for a homogeneous, almost complete global coverage of magnetic field data for the Earth. The more than six years worth of CHAMP geomagnetic field measurements, coupled with other orbiting missions, have paved the way for the development of global magnetic field models with unprecedented resolutions of the core, its secular variation and the lithosphere. Recent examples would include internal field models like CHAOS (Olsen et al., 2006), and static lithospheric field models such as MF4 and MF5 (Maus et al., 2006, 2007).

In this study, the quietest day of each month from 20012005 according to the list of international Q-days ${ }^{2}$ was considered. All the available CHAMP orbit and vector data in the NEC (North-East-Center, a local frame with a radial component pointing toward the center of the Earth and two others pointing North and East) coordinate frame from these $60 \mathrm{se}-$ lected days were used in this study. The list of International Q-days (Quiet-days) and D-days (Disturbed-days) is a ranking of the days in a particular month, for the purpose of clas-

\footnotetext{
${ }^{1}$ http://www.gfz-potsdam.de/pb1/op/champ/index_CHAMP. html

${ }^{2} \mathrm{http} / / /$ www.gfz-potsdam.de/pb2/pb23/niemegk/kp_index/ quietdst/
}

sifying them by their relative level of geomagnetic activity, based on the $K_{p}$ index. Each day is assigned an ordered rank on the basis of the sum, sum of squares, and the maximum of the eight daily $K_{p}$ values. The mean of these three rank values is computed and assigned as the overall rank for that day. The ten lowest ranks represent the Q-days and the five highest ranks represent the D-days. It is important to note that these ranks are only relative to other days in the same month and bare no relation to other months or years which may have vastly different absolute levels of geomagnetic activity (e.g., 8 January 2004 is the quietest day of its month but the $K_{p}$ for the day ranges as high as $3^{+}$).

\subsection{Models}

\subsubsection{CHAOS model}

Briefly stated (for a complete, detailed description please refer to the paper of Olsen et al., 2006) the CHAOS model describes the Earth's magnetic field over the timeframe of the current epoch. It models the geomagnetic field using spherical harmonics up to degree $n=50$ for the static field, $n=18$ for the first time derivative (secular variation), and $n=14$ for the quadratic and cubic time derivatives. The temporal variation of the core field is described using splines (for $n \leq 14$ ). It was developed using high-precision, geomagnetic measurements from the Ørsted, CHAMP and SAC-C satellite missions spanning over 6.5 years (including data between March 1999-December 2005).

\subsubsection{TIE-GCM}

The TIE-GCM on the other hand is a self-consistent, physicsbased simulation of neutral winds, conductivities, electric fields, various atmospheric species concentrations, and current densities having minimal direct observational input data. With only the highlights discussed herein, please consult the papers of Richmond et al. (1992) and Richmond (1995) for a proper treatment of this multifaceted model.

At the heart of the model, with regards to ionospheric electrodynamics, is the ionospheric wind dynamo, which is a process whereby thermospheric winds in the upper atmosphere transport the charged ions in the ionosphere through the geomagnetic field generating electric fields and currents (Richmond, 1989).

$$
\boldsymbol{J}=\sigma_{P}(\boldsymbol{E}+\boldsymbol{u} \times \boldsymbol{B})+\sigma_{H} \boldsymbol{b} \times(\boldsymbol{E}+\boldsymbol{u} \times \boldsymbol{B})+\boldsymbol{J}_{\|}+\boldsymbol{J}_{M}
$$

The Ohm's law expression for the current density from Richmond (1995) in Eq. (1) is used to solve for the electric field equatorward of $\pm 60^{\circ}$ magnetic latitude (imposed otherwise). In Eq. (1) $\boldsymbol{B}$ is the magnetic field with parallel unit vector $\boldsymbol{b}$, $\boldsymbol{E}$ is the electric field, $\boldsymbol{u}$ is the neutral wind velocity, and $\sigma_{P}$ and $\sigma_{H}$ are the Pedersen and Hall conductivities. Also included is a field aligned component of the current density $\boldsymbol{J}_{\|}$ and a non-ohmic, magnetospheric component $\boldsymbol{J}_{M}$. 
The International Geomagnetic Reference Field (IGRF/DGRF models) ${ }^{3}$ (Maus et al., 2005) is used for the purpose of casting the formulation into modified magnetic apex coordinates (Richmond, 1995), which is a more convenient coordinate frame for performing the calculations. As a simplifying measure, geomagnetic field lines are assumed to be equipotential, which reduces the electrodynamic equations to two dimensions. In order to ensure that the divergence of the total current vanishes it is assumed that field-aligned current flows between both hemispheres. Induced Earth currents are simulated assuming a perfectly conducting layer at a depth of $600 \mathrm{~km}$ below the Earth's surface. Height-integrated horizontal ionospheric currents are treated as currents in a thin shell at an altitude of $110 \mathrm{~km}$, connected to field-aligned currents. Atmospheric tides from the Global Scale Wave Model (GSWM) (Hagan and Forbes, 2002, 2003) can be used as a lower boundary condition and magnetic activity index values, like $K_{p}$ and $F_{10.7}{ }^{4}$, can be varied to simulate certain atmospheric conditions and configurations (Maute et al., 2003).

\section{Method of analysis}

The main steps in this analysis are the following: 1) The top ranked international Q-days from 2001-2005 are selected; 2) The input parameters for the TIE-GCM are established and the model is executed; 3 ) The magnetic perturbations are computed from the TIE-GCM output; 4) The corresponding CHAMP data are acquired and used with CHAOS to compute residuals; 5) The CHAMP/CHAOS residuals are compared with TIE-GCM magnetic perturbations. These steps are detailed in the remaining portion of this section.

1) Generally speaking, most core field modeling restricts its input datasets to include only data taken during local nighttime hours under conditions of low magnetic activity, in an effort to minimize external and other transient effects. For this reason it was decided to use only days with low geomagnetic activity for this initial comparison study since it is the most likely category of data to have immediate use in the modeling community. The selected dates in Table 1 represent the quietest day of each month, in terms of geomagnetic activity, from 2001-2005 as determined by the list of International Q-days. Figure 1 displays graphically the $K_{p}$ range and the $F_{10.7}$ for these dates.

2) The TIE-GCM was used to model each of the 60 quiet days from 2001 to 2005 listed in Table 1. The model permits its lower boundary condition to be specified by the GSWM; therefore, included were both the migrating (diurnal and semidiurnal) and non-migrating (diurnal and semidiurnal) atmospheric tides. The default integrated Heelis electric potential model (Heelis et al., 1974) was invoked. In

\footnotetext{
${ }^{3}$ http://www.ngdc.noaa.gov/IAGA/vmod/igrf.html

${ }^{4} \mathrm{ftp}$ ://ftp.gfz-potsdam.de/pub/home/obs/kp-ap/
}

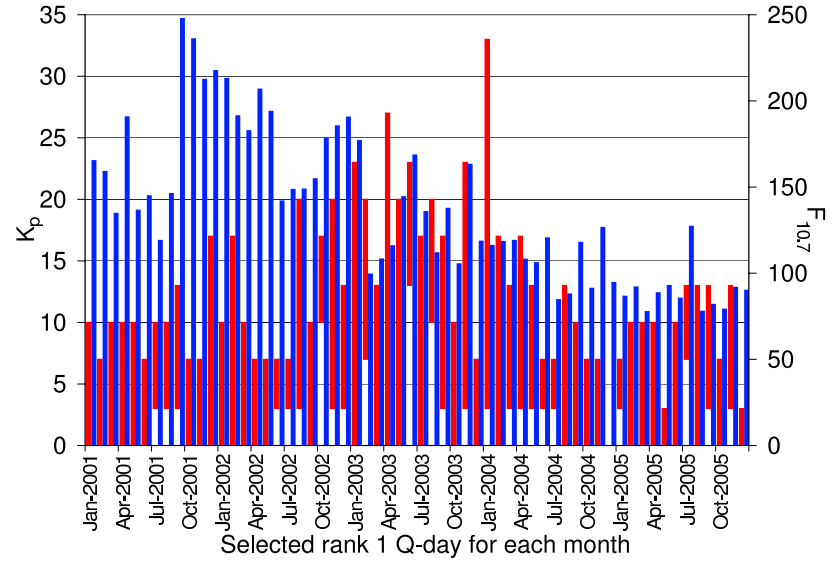

Fig. 1. Activity indices including the $K_{p}$ range (red) and $F_{10.7}$ (blue) for the selected rank 1 Q-days of each month.

addition, a two-day initial buffer was considered so as to allow the model to achieve an equilibrium state by the time the dates of interest were reached. The model internally calculates all quantities at every time-step ( $2 \mathrm{~min}$ ); however, only one time-step per modeled hour was considered due to data file size constraints.

In order to investigate the model's sensitivity, the following five cases of the input parameter $F_{10.7}$ (measured in solar flux units, $1 \mathrm{sfu}=10^{-22} \mathrm{~W} \mathrm{~m}^{-2} \mathrm{~Hz}^{-1}$ ) were used for each day: $F_{10.7}=70,90,150,190$ and GPI. Where GPI is a geophysical indices database of $K_{p}$ and $F_{10.7}$ used to compute their real-time values by interpolating and updating the model for every time-step, instead of keeping them fixed. $F_{10.7}$ is interpolated to the current model time-step using data from \pm 2 days, whereas $K_{p}$ is interpolated using a \pm 1 day window of its eight daily 3 -h values. A subsequent investigation varied instead the $K_{p}$ values for a subset of the selected days.

With the above indicated input parameters, the TIE-GCM model is executed. Figure 2 represents a characteristic example of the TIE-GCM prediction of the height-integrated horizontal current density for 14 February 2002 using realtime GPI inputs rather than fixed activity index values for the eastward component. The Equatorial Electro-Jet (EEJ) signature has been reproduced in the eastward component with amplitude of around $0.15 \mathrm{~A} / \mathrm{m}$.

3) The calculation process for magnetic perturbations involves making a few assumptions (Richmond, 2002). As noted before, the height-integrated horizontal ionospheric currents are treated as currents in a thin shell located at an altitude of $110 \mathrm{~km}$, and connected to field-aligned currents. The field-aligned currents are treated as though they are flowing on dipolar field lines, while zonal currents in the magnetosphere are ignored. Calculated ground magnetic effects are used to define equivalent horizontal ionospheric currents in a 
Table 1. The selected dates used in this comparison study represent the quietest day of each month from 2001-2005 as determined by the list of International Q-days. Starred dates are the quietest in a given year.

\begin{tabular}{|c|c|c|c|c|}
\hline $\begin{array}{l}\text { Date } \\
(2001)\end{array}$ & $\begin{array}{l}\text { Date } \\
(2002)\end{array}$ & $\begin{array}{l}\text { Date } \\
(2003)\end{array}$ & $\begin{array}{l}\text { Date } \\
(2004)\end{array}$ & $\begin{array}{l}\text { Date } \\
(2005)\end{array}$ \\
\hline 1 Jan 2001 & 3 Jan 2002 & 9 Jan 2003 & 8 Jan 2004 & 26 Jan 2005 \\
\hline 3 Feb 2001 & 14 Feb 2002 & 25 Feb 2003 & 26 Feb 2004 & 5 Feb 2005 \\
\hline 15 March 2001 & 17 March 2002 & 25 March 2003 & 24 March 2004 & 4 March 2005 \\
\hline 30 April 2001 & 8 April 2002 & 7 April 2003 & 2 April 2004 & 10 April 2005 \\
\hline 31 May 2001 & 24 May $2002 \star$ & 4 May 2003 & 26 May 2004 & 26 May 2005 \\
\hline 28 June $2001 \star$ & 28 June 2002 & 12 June 2003 & 22 June 2004 & 21 June 2005 \\
\hline 28 July 2001 & 14 July 2002 & 8 July 2003 & 8 July 2004 & 6 July 2005 \\
\hline 16 Aug 2001 & 6 Aug 2002 & 31 Aug 2003 & 4 Aug 2004 & 11 Aug 2005 \\
\hline 10 Sep 2001 & 23 Sep 2002 & 28 Sep 2003 & 11 Sep 2004 & 24 Sep 2005 \\
\hline 24 Oct 2001 & 13 Oct 2002 & 11 Oct 2003 & 17 Oct 2004 & 15 Oct 2005 \\
\hline 3 Nov 2001 & 8 Nov 2002 & 28 Nov 2003 & 6 Nov 2004 & 16 Nov 2005 \\
\hline 9 Dec 2001 & 18 Dec 2002 & 19 Dec $2003 \star$ & 4 Dec $2004 \star$ & 23 Dec $2005 \star$ \\
\hline
\end{tabular}

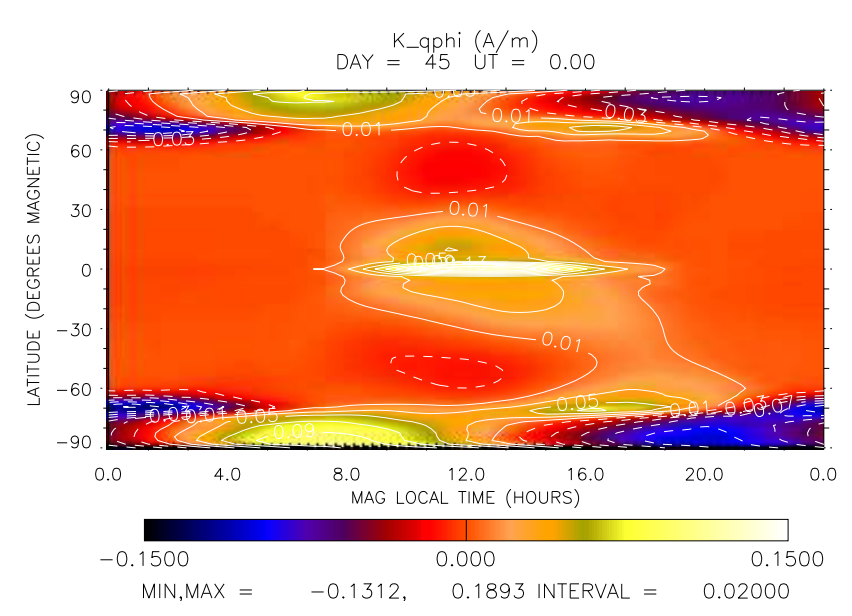

Fig. 2. The eastward component of the height-integrated horizontal current density in units of A/m predicted by the TIE-GCM for 14 February 2002 using GPI inputs.

shell at a height of $110 \mathrm{~km}$ (Maute et al., 2002). A satellite altitude of $430 \mathrm{~km}$ is assumed for this calculation.

A thin shell of height-integrated horizontal ionospheric current density is described in Richmond (1995) as

$\boldsymbol{K}=K_{q \phi} \boldsymbol{f}_{\mathbf{1}}+K_{q \lambda} \boldsymbol{f}_{\mathbf{2}}$

with basis functions $\boldsymbol{f}_{\mathbf{1}}$ and $\boldsymbol{f}_{\mathbf{2}}$ and northward $\left(K_{q \phi}\right)$ and eastward $\left(K_{q \lambda}\right)$ directional components, which are calculated by the TIE-GCM (e.g. Fig. 2).

These components are used to compute an equivalent current function discussed in Richmond (1974). The equivalent current system is a non-physical current sheet, yet still has the capability of reproducing the same ground magnetic perturbations as does the true three dimensional current system. The difference between this equivalent current function and

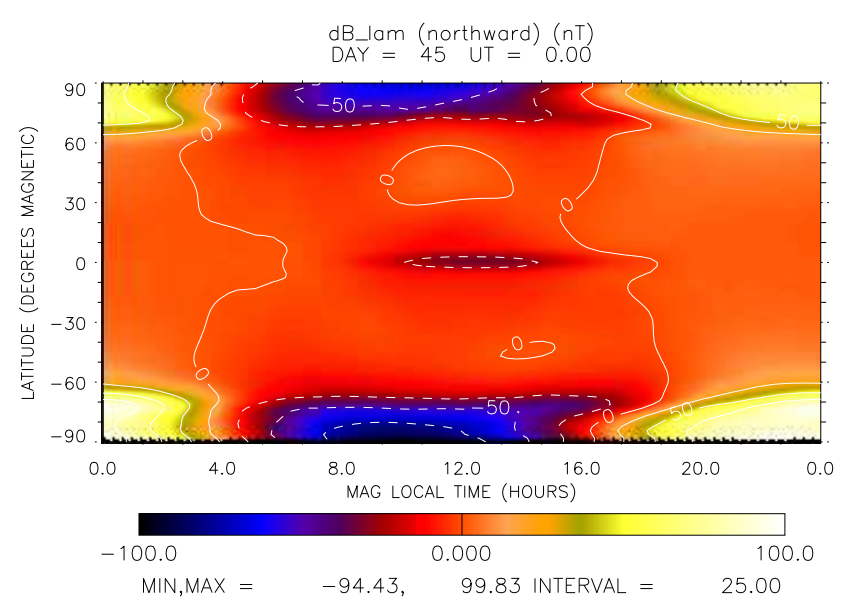

Fig. 3. The northward component of the magnetic perturbation in units of nT predicted by the TIE-GCM for 14 February 2002 using GPI inputs.

$K_{q \phi}$ and $K_{q \lambda}$ yields the unequivalent current function. Each of these two current sheets are used in the magnetic perturbation computation at satellite altitude. The equivalent current function can be expressed as an expansion in spherical harmonic coefficients, which are then used to calculate the magnetic potential. From magnetic potential it is straightforward to compute the magnetic field perturbations.

Figure 3 represents the northward component at satellite altitude of the magnetic perturbation, produced by postprocessing the TIE-GCM predictions of current densities, for 14 February 2002 using real-time GPI inputs. The EEJ signature seen in the eastward component of the height-integrated current density produces a negative magnetic perturbation, of order $-30 \mathrm{nT}$, in the northward magnetic perturbation component. 
4) The TIE-GCM magnetic perturbations reproduce the fields arising from the modeled current sheet, and so represent only external sources. In order to create an approximate analogue of this using geomagnetic satellite data, which is a combination of many sources, residuals are computed. CHAMP vector data was acquired for the dates listed in Table 1 and residuals were then computed using the full CHAOS model in order to remove core and crustal contributions.

5) Direct comparisons of the CHAMP/CHAOS residuals and the TIE-GCM perturbations are made on an orbit by orbit basis for each day. This is done by interpolating the 24 hourly perturbation maps at the spacecraft's time and position. Because of the rather course temporal resolution, small scale jumps can occur at the junction of two temporally adjacent perturbation maps. A subtraction between the CHAMP/CHAOS residuals and the TIE-GCM perturbation is computed, from which the misfit (mean and absolute mean deviation) is deduced. This misfit is then ranked relative to the various input parameters for each orbit and for the daily average.

\section{Results}

The results presented here were obtained by varying different parameters in the TIE-GCM magnetic perturbation computation and are detailed in the remainder of the section. These computations are then used to evaluate their effectiveness in a main field modeling situation.

4.1 Effects of $F_{10.7}$ and $K_{p}$ on the TIE-GCM magnetic perturbation predictions

The main thrust so far has been to do a systemic comparison of the effects of varying $F_{10.7}$ on the TIE-GCM magnetic perturbation prediction and to see how it relates to observed data. To this end, the quietest magnetically active day (as discussed earlier) of each month from 2001-2005 has been used for the TIE-GCM calculation. For each day the model was executed using the GPI and then again with fixed $F_{10.7}$ magnetic activity inputs equal to $F_{10.7}=70,90,150$ and 190 . Also held fixed were the cross-tail potential (CTPOTEN=45) and the hemispheric power (POWER=16), which are parameterized in the model to be functions of $K_{p}$,

POWER $=\operatorname{MAX}\left(3.0,-2.78+9.33 K_{p}^{i}\right)$

CTPOTEN $=29.0+11.0 K_{p}^{i}$,

where $K_{p}^{i}$ is the interpolated $K_{p}$ value for the particular time step.

The TIE-GCM magnetic perturbation predictions (Fig. 3) were compared to the CHAMP/CHAOS residuals along the orbit-track in order to produce Fig. 4. The full CHAOS model was used to compute residuals for the corresponding CHAMP vector measurements. Plotted also is a curve representing the residual calculated from the difference of the CHAMP/CHAOS residual and the predicted TIEGCM magnetic perturbation. The CHAMP/CHAOS and CHAMP/CHAOS/TIE-GCM residuals have had their mean subtracted so as to remove their offset from zero and bring all datasets to a common level (see MEAN statistic). For this individual plot, the CHAMP satellite was in approximately a 10:00 a.m. solar local time (SLT) plane. In the top and bottom left-hand corners are displayed the absolute mean deviation, mean and area under the curve in the following format: [MDEV(MEAN)-AREA]. The MEAN represents an important quantity in terms of the overall offset. Ideally, a CHAMP/CHAOS residual MEAN would be zero, indicating that the model and the data are of equivalent amplitude. However, this is not the case because of noise, as well as unresolved and unmodeled phenomena mainly in CHAOS. Instead an offset is observed, which ideally the TIE-GCM perturbation can partially ameliorate. The MDEV is also important in that it is a measure of the variation in the time series. A residual MDEV of zero would correspond to a horizontal line and indicate that the CHAMP and CHAOS exactly mirror each other in terms of small scale variations. Again from a practicality standpoint this is not possible and is evidenced by the EEJ signatures visible in the CHAMP/CHAOS residuals because CHAOS does not model such systems. The TIEGCM, however, does model the EEJ and so if an accurate magnetic perturbation prediction can be computed and then subtracted out, a reduction in the EEJ signature should be visible, which in turn would reduce (all things being equal) the MDEV value. The AREA statistic is a measure of the area under the residual time-series and so is an amalgamation of the MEAN and MDEV, but can be heavily skewed by a large MEAN value.

The EEJ signature seen in $B_{\theta}$ (Fig. 4c) for this particular orbit pass is fairly well reproduced in location, even though the amplitude is quite a bit smaller. After the application of the TIE-GCM magnetic perturbation prediction, this component yields both a reduction in MDEV (spread) and MEAN (offset), likewise for the total field (Fig. 4a). In this case the run with the $F_{10.7}$ held fixed at 190 seems to have produced the best overall fit (the actual $F_{10.7}$ for this date was 191.3); however, it is not always the case that the actual values produces the best result. The plot window for the $B_{r}$ component (Fig. 4b) shows the ability of the TIE-GCM to reproduce the low/high transition centered around the magnetic equator associated with Sq, however its poorer job in the tail regions has it netting a worse overall MDEV and MEAN. The $B_{\phi}$ component (Fig. 4b) has a mixed result whereby the model matched a slight trend but the amplitude was off causing a better MDEV but worse MEAN. But overall Fig. 4 actually represents a relatively good fit, whereas many individual day comparisons can show more inconsistent results.

Figure 5 is a summary plot of Fig. 4 (corresponding to orbit number 3) across all orbits in the day. In Fig. 5a the TIEGCM models generally show an improvement by reducing 


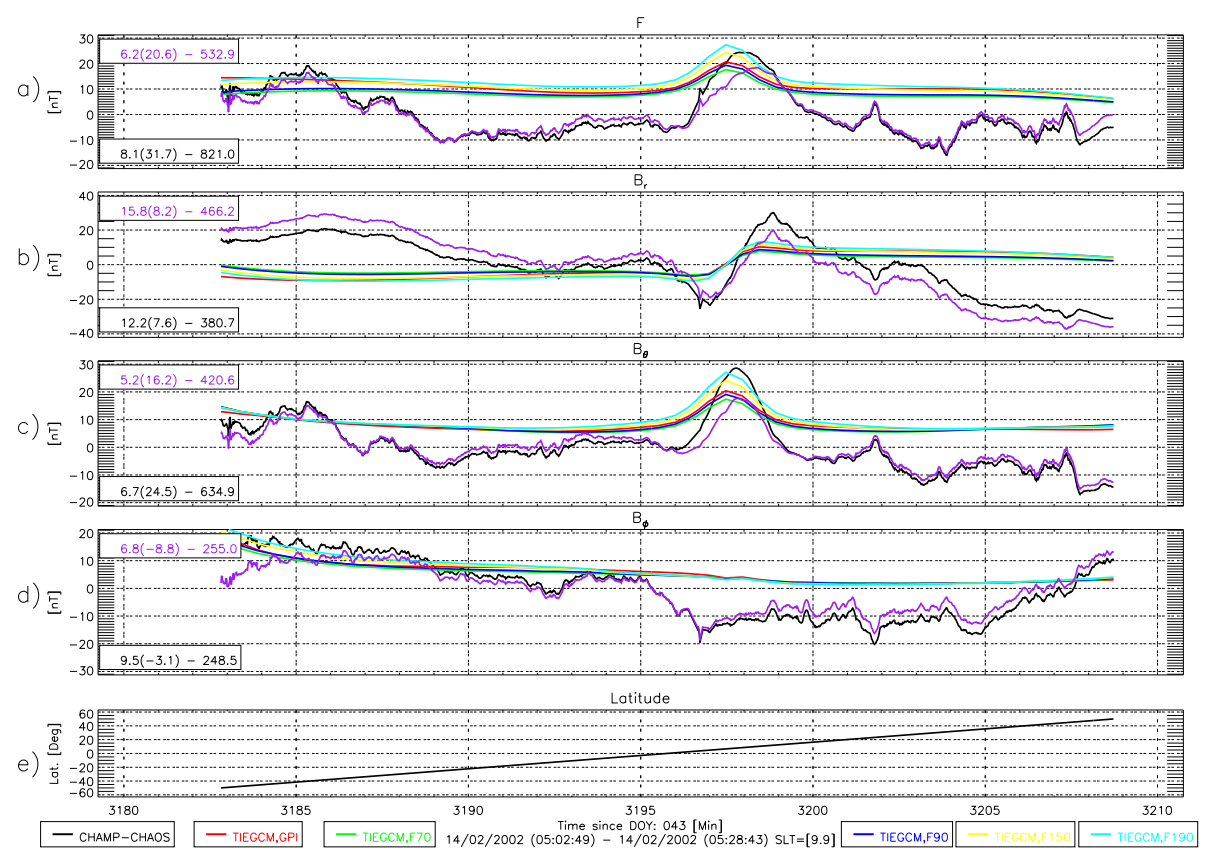

Fig. 4. The residual and TIE-GCM perturbation prediction data along the satellite orbit track for one orbit pass on 14 February 2002 between $\pm 50^{\circ}$ latitude in units of nT. The bottom axis is time (minutes) since the start of the modeling run, in this case 12 February 2002 . The plot windows correspond to (a) the total field perturbation, (b) the $B_{r}$ component of the perturbation, (c) the $B_{\theta}$ component, (d) the $B_{\phi}$ component, and (e) the satellite latitude. All five TIE-GCM model runs are shown vs. the CHAMP data and perturbation residuals using the following color scheme: $F_{10.7}=\mathrm{GPI}$ (red), $F_{10.7}=70$ (green), $F_{10.7}=90$ (blue), $F_{10.7}=150$ (yellow), $F_{10.7}=190$ (cyan), CHAMP/CHAOS residual (black), and CHAMP/CHAOS/TIE-GCM(GPI) residual (purple).

the MDEV and MEAN for the $B_{\theta}$ component. Though not displayed, the total field $B_{F}$ shows a similar character. As is typical, the $B_{r}$ (Fig. 5b) and $B_{\phi}$ (not shown) components are more inconsistent. It is hard to draw anything conclusive, but overall the TIE-GCM models seem to bring an improvement in the final residuals.

In an effort to better characterize the TIE-GCM model results in relation to CHAMP data, the quietest day of each year from 2001-2005 was selected for further study in varying the $K_{p}$ index. These days (starred dates in Table 1) were selected by first examining the $K_{p}$ range for each of the days in Table 1, then selecting the days with the lowest value for the high end $K_{p}$ value. In case of duplicates the day with the smaller $F_{10.7}$ value was selected.

In this particular test only constant values of $K_{p}$ were used and were varied from $0-8$ with a fixed $F_{10.7}$ for all runs. The model does not directly take $K_{p}$ as an input, but rather indirectly through the parameters POWER and CTPOTEN. The values of $0-8$ for $K_{p}^{i}$ were substituted into Eqs. (3-4) and the resulting values were used as the inputs to the TIEGCM model runs, in order to see the effects of changing just the $K_{p}$, while other parameters were held fixed.

Of the model runs plotted in Fig. 7, the best fit for the total field $\left(B_{F}\right)$ visually appears to be for $K_{p}=2$, which is close to the upper limit of the actual $K_{p}$ range of $0-2^{-}$. The next best fit corresponds to the curve for $K_{p}=1$, followed by the $K_{p}=0, K_{p}=4$, and $K_{p}=8$. However, it should be remembered that in Fig. 7 the CHAMP/CHAOS residual has had its mean removed so as to center the curve about zero, but the magnetic perturbation curves have not. The $B_{\phi}$ component shows better agreement in the MEAN and AREA with TIEGCM runs of smaller $K_{p}$ value. The agreement generally tends to degrade with increasing $K_{p}$. This also seems to be the trend seen in the MDEV of the $B_{\theta}$ component.

Curiously it appears that generally the $K_{p}=4$ is the best for the $B_{\theta}$ component in the MEAN and AREA statistic, probably because it tends to show the best approximation of the EEJ in terms of signal amplitude, without having the tail diverge as much as does the $K_{p}=8$ curve. The other components have no clear patterns.

\subsection{TIE-GCM as a possibility for correcting magnetic field data}

One prospect here is to see if the TIE-GCM can be used to partially correct dayside data so as to supplement the current geomagnetic datasets, especially during times and places where there are insufficient quiet nighttime data available. Using the same procedure as already described, the TIEGCM was used to compute analogous model predictions. 

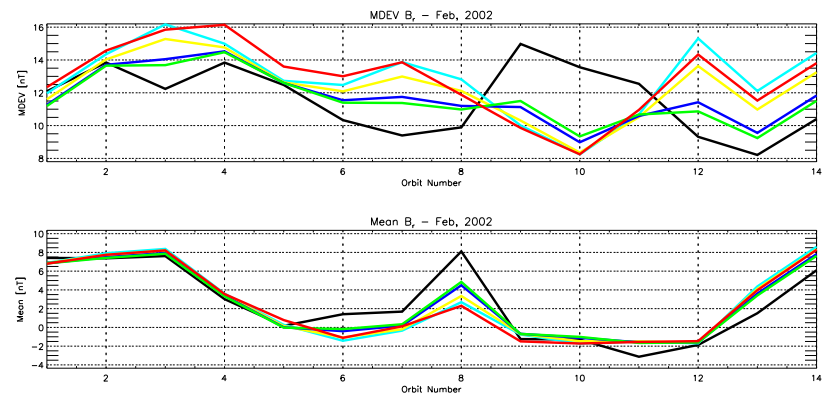

$a B_{r}-\mathrm{Feb}, 2002$

(a)
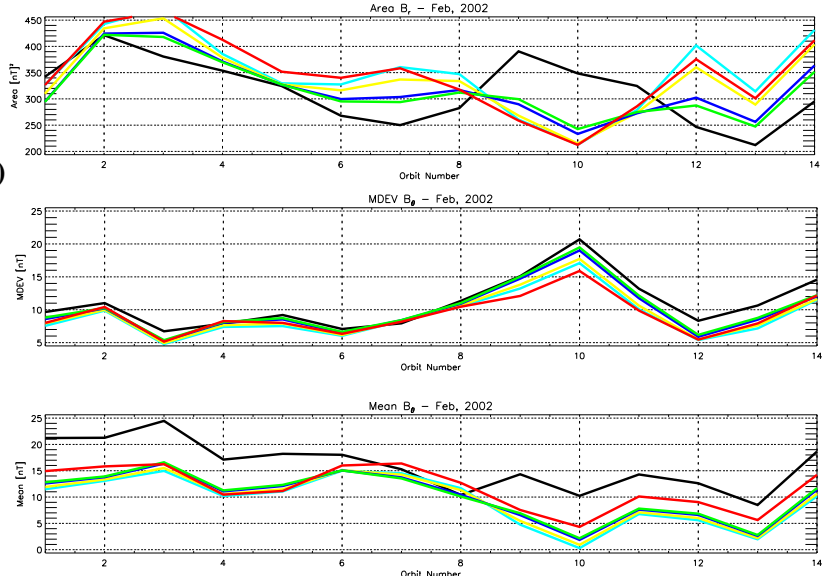

$B_{\theta}-F_{e b}, 2002$

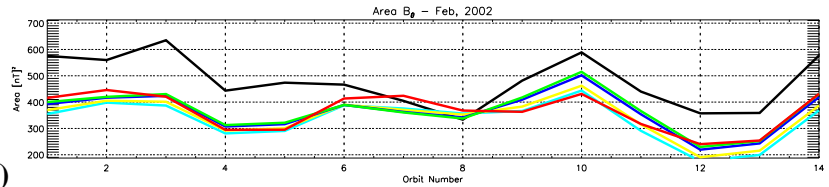

(a)
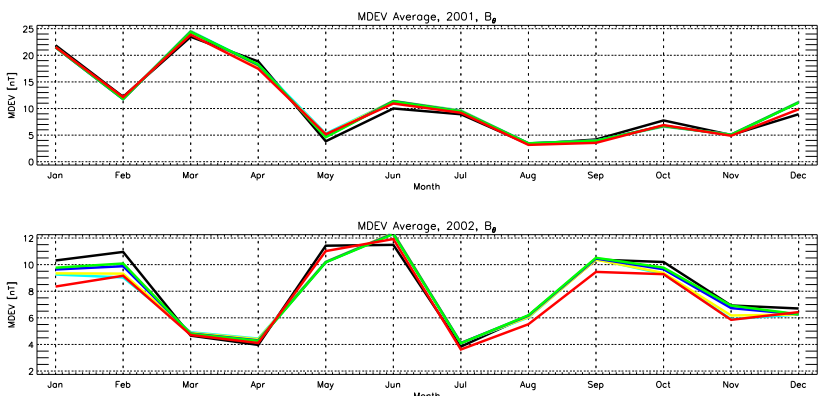

DEV Average, 2003, $\mathrm{B}_{\theta}$
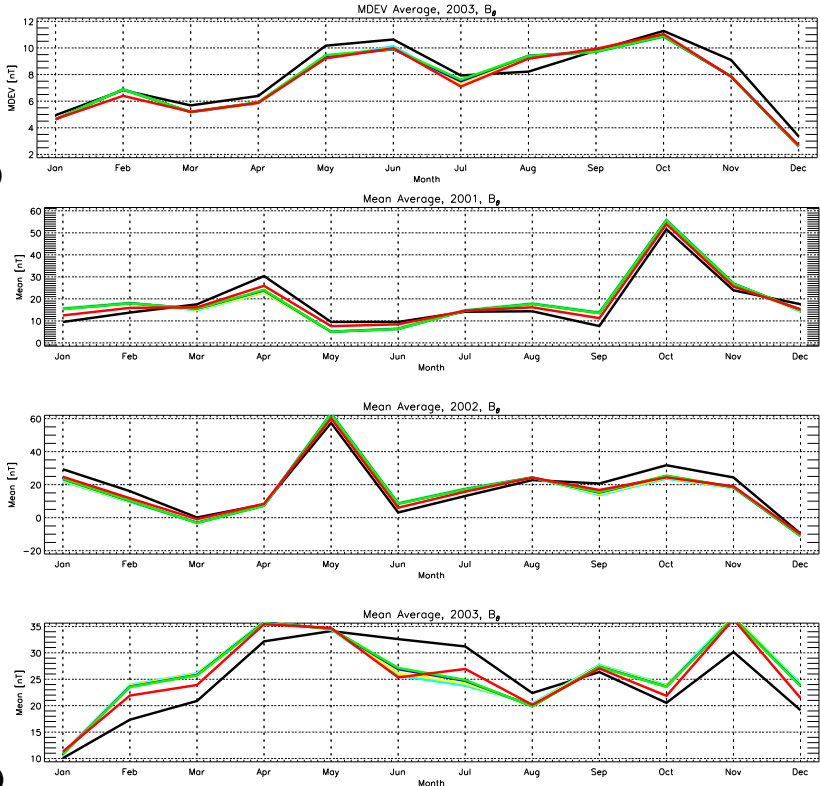

Fig. 5. Statistics of the dayside orbit passes for 14 February 2002: MDEV (top), MEAN (middle), and Area (bottom) vs. orbit number. The CHAMP/CHAOS/TIE-GCM (a) $B_{r}$ and (b) $B_{\theta}$ residuals as in Fig. 4 are shown vs. the orbit number. All five TIE-GCM model runs (GPI (red), $F_{10.7}=70$ (green), $F_{10.7}=90$ (blue), $F_{10.7}=150$ (yellow), $F_{10.7}=190$ (cyan)) and the CHAMP residuals (black) are displayed.

The difference between the dataset/model residuals and the TIE-GCM predictions were then computed to see if any of the statistical quantities could be improved. A main objective was to see if the TIE-GCM "correction" would reduce the residuals on the dayside to the same level as the residuals on the nightside. In this case two months of CHAMP data were used to develop models to characterize the usefulness of folding TIE-GCM corrected dayside data into modeling datasets. For this, five models were derived using the modeling techniques developed in Cain et al. (2003): a standard model with nightside data and no dayside data, a model with nightside data and uncorrected dayside data, and lastly three models with nightside data and dayside data corrected using the TIE-GCM with either GPI, $K_{p}=0$ or $K_{p}=8$ inputs. Only two months worth of data were used to artificially create a

Fig. 6. The time-series of the average orbit pass statistics (a) MDEV and (b) MEAN (nT vs. Month) for each of the selected days spanning the years 2001 (top), 2002 (middle), and 2003 (bottom) for the $B_{\theta}$ component. All five TIE-GCM model runs are represented (GPI (red), $F_{10.7}=70$ (green), $F_{10.7}=90$ (blue), $F_{10.7}=150$ (yellow), $F_{10.7}=190$ (cyan)) as well as the CHAMP residuals (black).

need for the use of dayside data; two months is enough to produce a reasonable model yet will still contain data coverage gaps (Fig. 8) that can be filled with dayside data. The five models are outlined herein:

\section{- Model 1}

CHAMP NEC vector and scalar data from $1 \mathrm{Au}-$ gust 2004-31 September 2004 was converted to geodetic $X, Y, Z$ and $F$ vector components. It was selected for local-times between 10:00 p.m.-02:00 a.m., $K_{p} \leq 1$, $F_{10.7} \leq 150$, and a $D_{s t}$ correction was applied. The vector data was limited to lie between $\pm 50^{\circ}$ magnetic latitude, while the scalar data was limited to be poleward of $\pm 50^{\circ}$ magnetic latitude. Residuals were computed using CHAOS to estimate data class weights and those data differing by more than $100 \mathrm{nT}$ were removed 


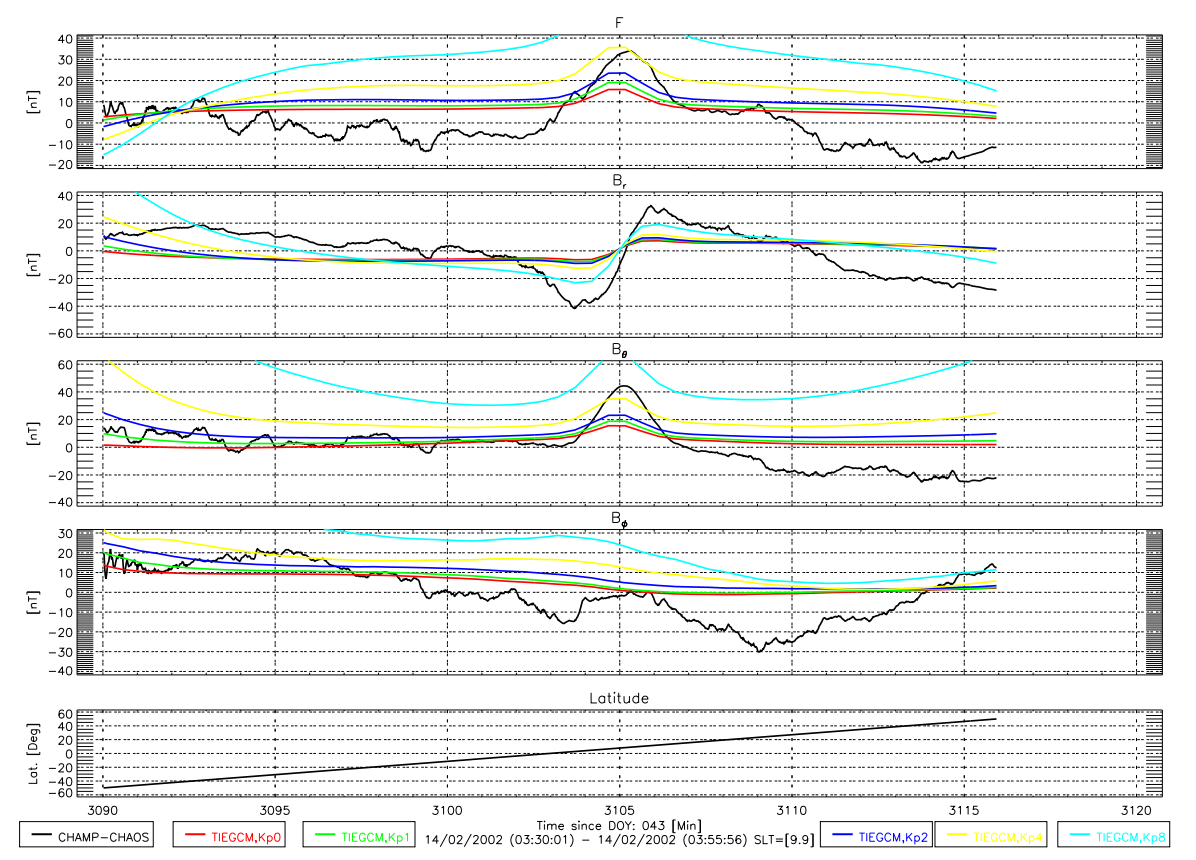

Fig. 7. The residual and TIE-GCM perturbation prediction data along a satellite orbit track for one dayside orbit pass on 14 February 2002 between $\pm 50^{\circ}$ latitude in units of $\mathrm{nT}$ comparing the effects of varying $K_{p}$. The bottom axis is time (minutes) since the start of the modeling run, in this case 12 February 2002. The plot windows correspond to (a) the total field perturbation, (b) the $B_{r}$ component of the perturbation, (c) the $B_{\theta}$ component, (d) the $B_{\phi}$ component, and (e) the satellite latitude (in degrees). A constant $F_{10.7}$ of 190 is used with either $K_{p}=0$ (red), $K_{p}=1$ (green), $K_{p}=2$ (blue), $K_{p}=4$ (yellow), $K_{p}=8$ (cyan), and CHAMP/CHAOS residual (black).

from the dataset. The entire dataset was equal-area shaved down to $\sim 200000$ data points and corresponding equal-area weight factors were determined. A truncated CHAOS model (limited to spherical harmonic degree $n=20$ with secular variation terms to degree $n=10$ ) was used as an initial state model.

\section{- Model 2}

This model is identical to Model 1 with the addition of dayside data. CHAMP vector data from $1 \mathrm{Au}-$ gust 2004-31 September 2004 was converted to geodetic $X, Y, Z$ and $F$ vector components. It was selected for local-times between 09:00 a.m.-03:00 p.m., $K_{p} \leq 1$, $F_{10.7} \leq 150$, and a $D_{s t}$ correction was applied. The vector data was restricted to lie between $\pm 50^{\circ}$ magnetic latitude. Residuals were computed using CHAOS to establish data class weights and those data differing by more than $100 \mathrm{nT}$ were removed from the dataset. The entire dataset was equal-area shaved down to $\sim 150000$ data points $(\sim 350000$ total $)$ and the corresponding equal-area weight factors were determined. A truncated CHAOS model (limited to spherical harmonic degree $n=20$ with secular variation terms to degree $n=10$ ) was used as an initial state model.

\section{- Models 3, 4 and 5}

These models are identical to Model 2 except the additional dayside data was further corrected using the TIE-GCM perturbations. Model 3 uses GPI TIE-GCM inputs, Model 4 uses fixed $K_{p}=0$ and $F_{10.7}=100$ inputs, and Model 5 uses fixed $K_{p}=8$ and $F_{10.7}=100$ inputs. A sample of the Model 3 correction is displayed in Fig. 9 for one orbit pass.

After running the models, residuals were computed between the resulting SHC and the input datasets. The error distribution of the residuals were fit to a Gaussian function and plotted in Fig. 10 with the results summarized in Table 2.

Comparing Models 1 and 2 shows that adding uncorrected dayside data increases the Gaussian width of the mid-latitude vector and polar scalar nightside data, which is not unexpected. The misfit for the nightside $B_{r}$ component data is larger by about $2 \mathrm{nT}$ (from $5.16 \mathrm{nT}$ to $7.38 \mathrm{nT}$ ), while the nightside $B_{\theta}$ component and the total field in the polar region increased by about $1 \mathrm{nT}$. The $B_{\phi}$ component showed only a modest gain in misfit. The TIE-GCM corrected dayside data in Model 3 reduces these misfits slightly for the $B_{\theta}$ and $B_{\phi}$ components, while the $B_{r}$ component misfit is dramatically reduced to be even smaller than that of Model 1, which altogether excludes dayside data. The low magnetic activity TIE-GCM correction used in Model 4 shows similar results 
Table 2. Gaussian fit parameters of the residual error distributions for Models 1-5.

\begin{tabular}{|c|c|c|c|c|c|c|c|c|c|}
\hline \multirow{2}{*}{ Class } & \multirow{2}{*}{ Model } & \multicolumn{2}{|c|}{$\mathrm{B}_{r}$} & \multicolumn{2}{|c|}{$\mathrm{B}_{\theta}$} & \multicolumn{2}{|c|}{$\mathrm{B}_{\phi}$} & \multicolumn{2}{|c|}{ B } \\
\hline & & Cen & Wid & Cen & Wid & Cen & Wid & Cen & Wid \\
\hline \multirow{5}{*}{ Night/Mid/Vector } & Model 1 & -5.43 & 5.16 & -0.12 & 3.70 & 1.88 & 3.16 & - & - \\
\hline & Model 2 & -5.87 & 7.38 & 0.75 & 4.99 & 2.12 & 3.31 & - & - \\
\hline & Model 3 & -5.82 & 5.07 & 1.23 & 4.73 & 2.10 & 3.24 & - & - \\
\hline & Model 4 & -5.85 & 5.10 & 1.11 & 4.76 & 2.10 & 3.23 & - & - \\
\hline & Model 5 & -5.30 & 7.39 & 5.43 & 5.00 & 1.86 & 3.34 & - & - \\
\hline \multirow{5}{*}{ Night/Pole/Scalar } & Model 1 & - & - & - & - & - & - & 0.70 & 7.43 \\
\hline & Model 2 & - & - & - & - & - & - & 1.40 & 8.78 \\
\hline & Model 3 & - & - & - & - & - & - & 0.84 & 7.92 \\
\hline & Model 4 & - & - & - & - & - & - & 0.93 & 8.06 \\
\hline & Model 5 & - & - & - & - & - & - & 0.22 & 9.99 \\
\hline \multirow{5}{*}{ Day/Mid/Vector } & Model 1 & - & - & - & - & - & - & - & - \\
\hline & Model 2 & 5.74 & 8.39 & -2.67 & 9.92 & -1.24 & 15.28 & - & - \\
\hline & Model 3 & 7.23 & 8.49 & -5.00 & 9.89 & 3.74 & 12.97 & - & - \\
\hline & Model 4 & 7.10 & 8.32 & -4.46 & 9.79 & 3.77 & 13.45 & - & - \\
\hline & Model 5 & 9.46 & 12.98 & -25.94 & 13.88 & -2.67 & 21.72 & - & - \\
\hline
\end{tabular}

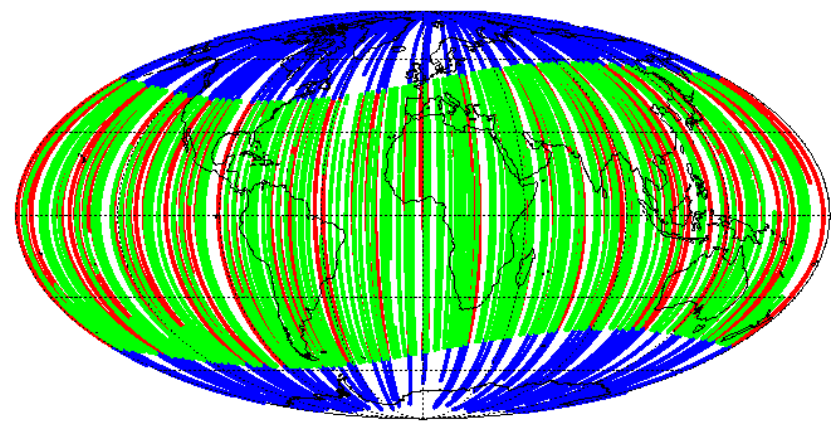

(a)Model 3

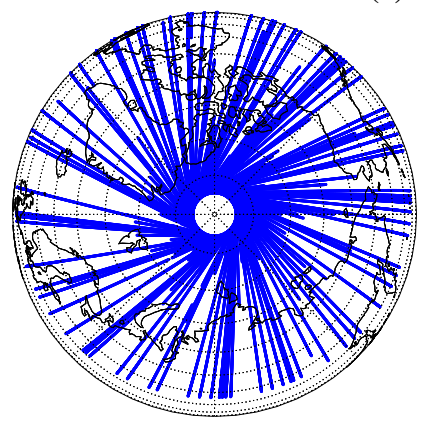

(b) North Pole

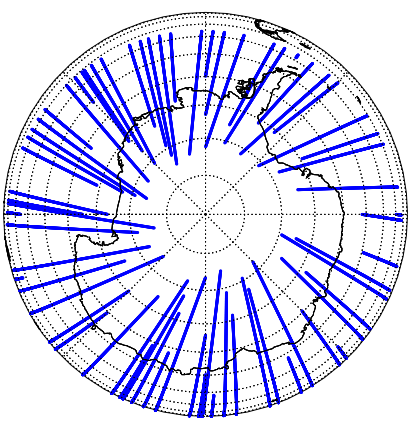

(c) South Pole
Fig. 8. Data distribution for Model 3 where red is CHAMP vector nightside, blue is CHAMP scalar nightside, and green is CHAMP vector dayside. Windows (b) and (c) show the scalar data distribution in the polar regions and demonstrate, especially in the southern polar region, the problem of using such a short time span.
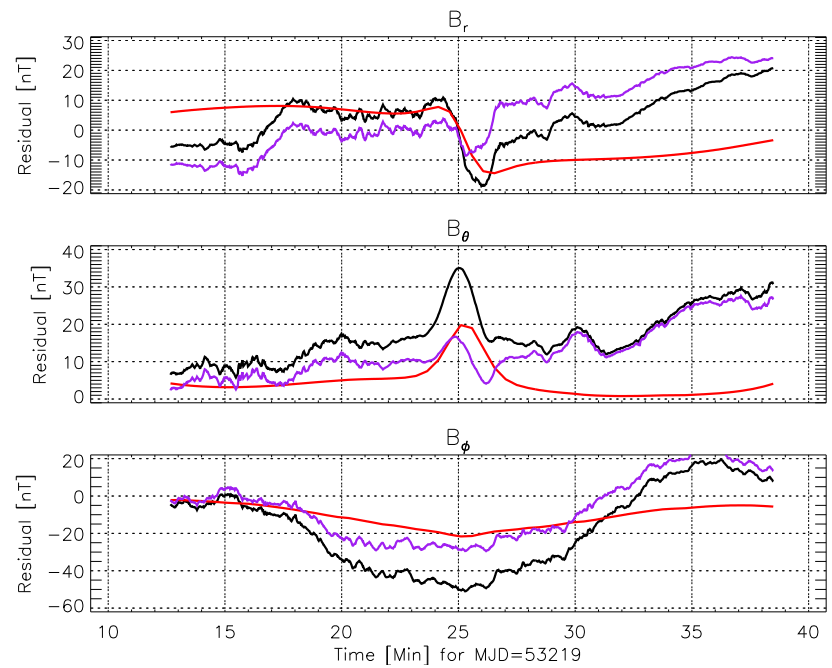

Fig. 9. The TIE-GCM correction (red) applied to a sample orbit of the dayside data in Model 3 for $B_{r}$ (top), $B_{\theta}$ (middle) and $B_{\phi}$ (bottom) components in nT vs. time [min]. The residual (black) of the dayside data was computed relative to the CHAOS model (residuals used to facilitate clarity). The difference of the data residual and the TIE-GCM correction is characterized by the purple curve and represents the data used as input to Model 3 (minus the CHAOS residual computation). While the TIE-GCM correction mirrors the data in a general sense, it can also adversely affect the corrected data by amplifying the misfit in some area (especially the higher latitude regions). 


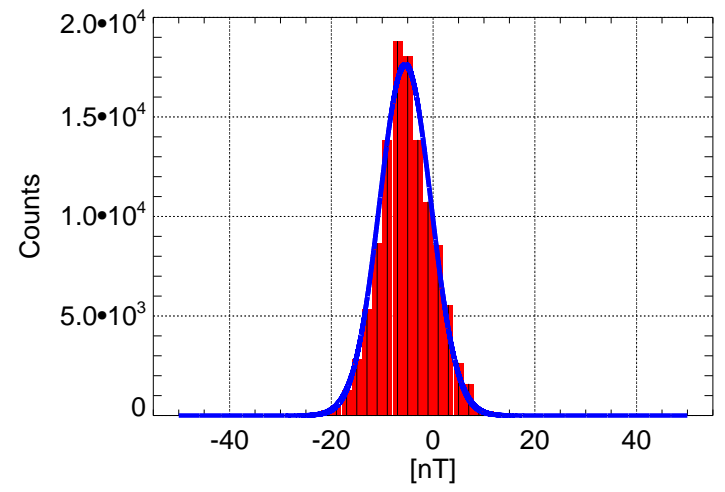

(a) Model $1-B_{r}$ Nightside Error

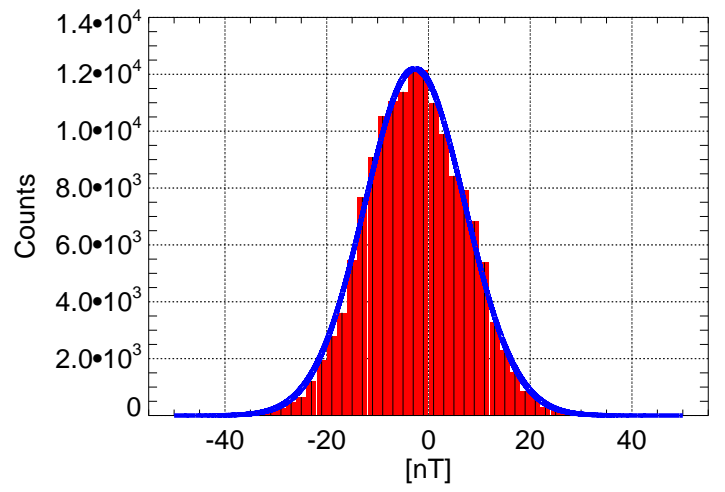

(b) Model $2-B_{\theta}$ Dayside Error

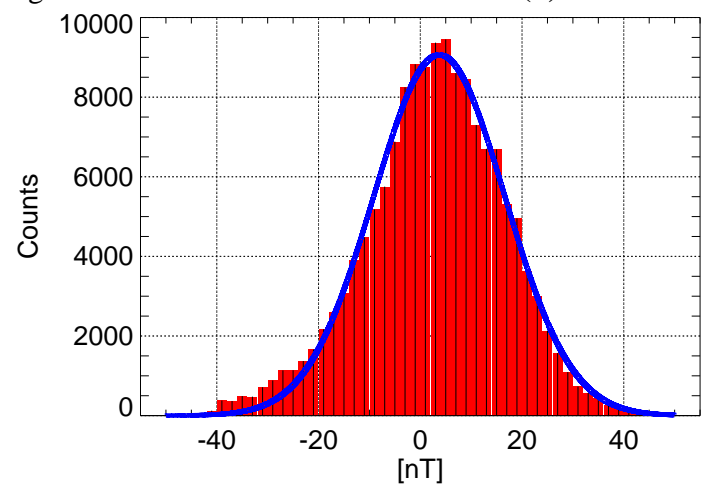

(c) Model $3-B_{\phi}$ Dayside Error

Fig. 10. A sampling of the error distributions with Gaussian fits for different model components in units of counts per $2 \mathrm{nT}$ bin.

to that of Model 3 with generally slightly higher fit errors, although the nightside $B_{r}$ is still lower than Model 1. The high magnetic activity TIE-GCM correction associated with Model 5 shows a level of misfit on par with that of Model 2 (uncorrected dayside).

The absolute misfit for mid-latitude dayside data is, of course, higher than that of the corresponding nightside data (greater by a factor anywhere from $1.5 \times$ for $B_{r}$ to $5 \times$ for $B_{\phi}$ ), but that is consistent with the expected higher noise characteristic of dayside measurements. However, the data show similar misfits in each component across Models 2, 3 and 4 with Model 4 having marginally better misfits in the $B_{r}$ and $B_{\theta}$ components and Model 3 having the best $B_{\phi}$ component misfit. Model 5 consistently demonstrates the highest misfit for each component, roughly $65 \%$ higher than the other models.

The differences between Model 2 and Model 3 shown in Figs. 11g-i reveal that the largest and most noticeable misfit appears in the sparsely sampled southern polar region, which unfortunately is unavoidable with a dataset of such limited temporal extent. The differences elsewhere, however, are fairly diffuse, although they seem to be associated with the orbit track lines. One of the stronger differences is located off the western coast of Central and South America.

\section{Conclusions}

Finally, for this initial study, one can see that the TIE-GCM can, to some degree, reproduce the residuals computed from CHAMP geomagnetic vector data. However, the quality of the improvement is rather inconsistent, sometimes the improvements being noticeable, sometimes not. This is evidenced, for example, in the mean average of the $B_{\theta}$ component for 2003 (Fig. 6), where there is a marked improvement from May until September, but not for the other months. For that same component however, the MDEV is consistently better for the years 2002 and 2001, but not in $B_{F}$.

But overall, the fact that a model derived using no in-situ magnetic data can match features in satellite data is promising. This modeling approach better lends itself to an understanding of the physics of the ionospheric sources than would a purely parameterized model. While the TIE-GCM requires substantial computational resources (although not overly so, the level of resolution and hardware used in this study required roughly one hour per modeled day), the results suggest that under the right circumstances it might be possible to use the TIE-GCM to pre-process dayside satellite data in order to supplement geomagnetic modeling efforts, especially during stretches when the availability of quality nighttime data is insufficient. This is underscored by the fact 


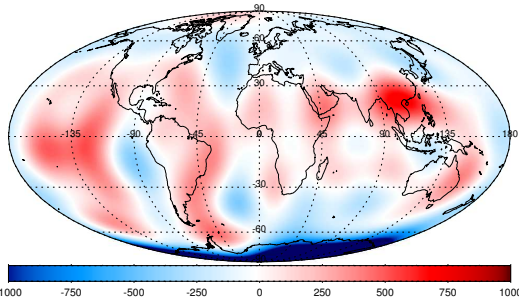

(a) $B_{r}$ : Model 1-Model 2

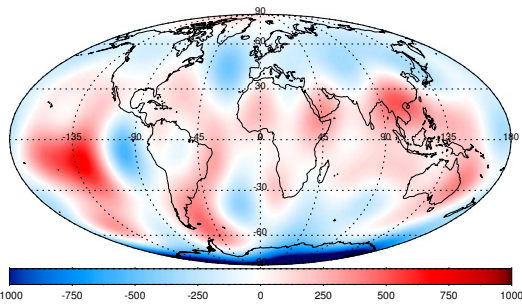

(d) $B_{r}$ : Model 1-Model 3

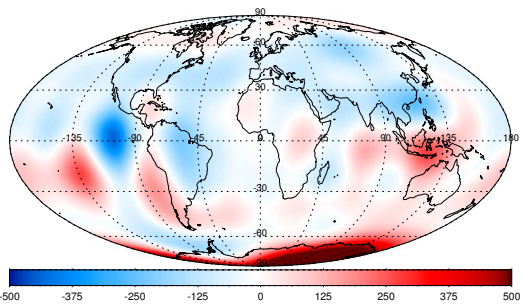

(g) $B_{r}:$ Model 2-Model 3

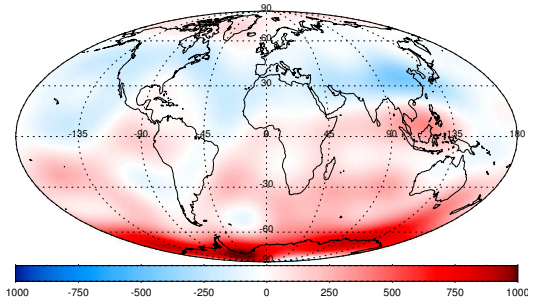

(b) $B_{\theta}$ : Model 1-Model 2

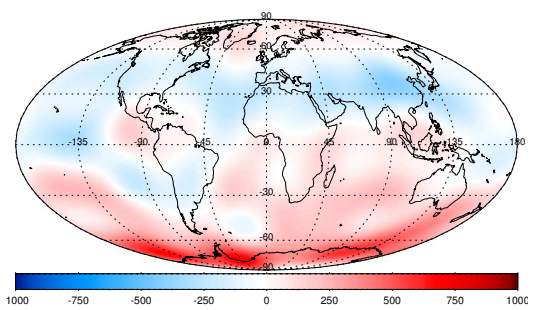

(e) $B_{\theta}$ : Model 1-Model 3

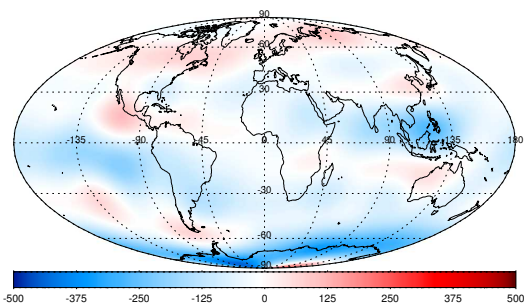

(h) $B_{\theta}$ : Model 2-Model 3

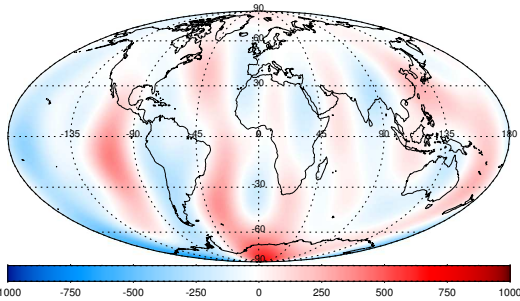

(c) $B_{\phi}$ : Model 1-Model 2

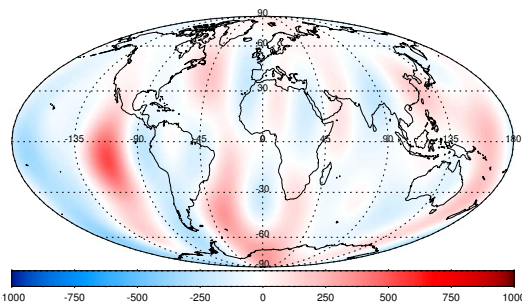

(f) $B_{\phi}:$ Model 1-Model 3

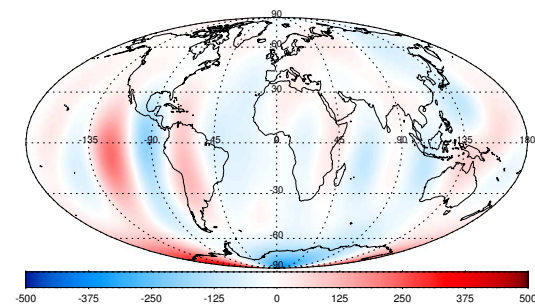

(i) $B_{\phi}$ : Model 2-Model 3

Fig. 11. Difference plots for Model 1-Model 2 (a-c, $\pm 1000 \mathrm{nT})$, Model 1-Model 3 (d-f, $\pm 1000 \mathrm{nT})$, and Model 2-Model 3 (g-i, $\pm 500 \mathrm{nT})$. Model 2 (uncorrected dayside data) and Model 3 (TIE-GCM corrected dayside data) show the largest difference in the southern polar region.

that Model 3, which includes corrected dayside data, has misfits both generally superior to Model 2 (uncorrected dayside data) and comparable with Model 1 (nighttime data only).

Part of the goal of such studies is to help sort out the sources of the magnetic field components that come from the crust, core, ionosphere, magnetosphere, induced, etc., by examining the observational data. This is important because while most modeling uses primarily nighttime data, it is conceivable that there are internal currents on the nightside that are induced from the dayside, which can enter such models and be misidentified as internal to the shell of spacecraft measurements. As geomagnetic modeling progresses this question will need to be addressed, and while it is unresolvable from this approach at the current stage, it remains a significant goal.

The forthcoming SWARM mission (Friis-Christensen et al., 2006), when coupled with the current complement of satellites, will make it possible to undertake a thorough investigation of a complete solar cycle. Other possible avenues of study include better pinning-down under what conditions the model best predicts the data. To proceed in this direction it is important to expand the tested parameter space to include inputs other than just $F_{10.7}, K_{p}$ and temporal range.
These would include varying TIE-GCM inputs like the atmospheric tides and the electric potential model. Also, broadening the selection criteria to include alternative methods of data selection like those based on the wavelet power spectrum (Schachtschneider et al., 2006), or conversely it may prove informative to purposely include days with more elevated geomagnetic activity. These new directions will be investigated in future work.

Acknowledgements. This work was partially supported by the FSU School for Computational Science and Information Technology (CSIT), by a grant of resources on the IBM pSeries 690 Power4based supercomputer "Eclipse". We would like to thank members of the High Altitude Observatory, including A. Richmond, A. Maute, and B. Foster, for access to and instruction in the use of the TIE-GCM and its associated programs.

Topical Editor M. Pinnock thanks A. W. P. Thomson and another anonymous referee for their help in evaluating this paper.

\section{References}

Cain, J. C., Mozzoni, D. T., Ferguson, B. B., and Ajayi, O.: Geomagnetic secular variation 1995-2000, J. Geophys. Res., 108(B3), 2161, doi:10.1029/2001JB001218, 2003. 
Friis-Christensen, E., Lühr, H., and Hulot, G.: Swarm: A constellation to study the Earth's magnetic field, Earth Planets Space, 58, 351-358, 2006.

Hagan, M. E. and Forbes, J. M.: Migrating and nonmigrating diurnal tides in the middle and upper atmosphere excited by tropospheric latent heat release, J. Geophys. Res., 107(D24), 4754, doi:10.1029/2001JD001236, 2002.

Hagan, M. E. and Forbes, J. M.: Migrating and nonmigrating semidiurnal tides in the upper atmosphere excited by tropospheric latent heat release, J. Geophys. Res., 108(A2), 1062, doi:10.1029/2002JA009466, 2003.

Heelis, R. A., Kendall, P. C., Moffett, R. J., Windle, D. W., and Rishbeth, H.: Electrical coupling of the E- and F-regions and its effect on F-region drifts and winds, Planet. Space Sci., 22(5), 743-756, 1974.

Maus, S., Macmillan, S., Chernova, T., Choi, S., Dater, D., Golovkov, V., Lesur, V., Lowes, F., Lühr, H., Mai, W., Mclean, S., Olsen, N., Rother, M., Sabaka, T., Thomson, A., and Zvereva, T.: The 10th-Generation International Geomagnetic Reference Field, Geophys. J. Int., 161, 561-565, doi:10.1111/j.1365246X.2005.02641.x, 2005.

Maus, S., Rother, M., Hemant, K., Stolle, C., Lühr, H., Kuvshinov, A., and Olsen, N.: Earth's lithospheric magnetic field determined to spherical harmonic degree 90 from CHAMP satellite measurements, Geophys. J. Int., 164, 319-330, doi:10.1111/j.1365246X.2005.02833.x, 2006.

Maus, S., Lühr, H., Rother, M., Hemant, K., Balasis, G., Ritter, P., and Stolle, C.: Fifth-generation lithospheric magnetic field model from CHAMP satellite measurements, Geochem. Geophys. Geosyst., 8, Q05013, doi:10.1029/2006GC001521, 2007.

Maute, A. I., Richmond, A. D., Hagan, M. E., and Roble, R. G.: Ionospheric Dynamo Currents and Magnetic Perturbations at the Ground and Above the Ionosphere Modeled by the TIEGCM, American Geophysical Union - Fall Meeting, San Francisco, USA, 6-10 December 2002, SA72B-0525, 2002.
Maute, A., Richmond, A., Sabaka, T., and Olsen, N.: Comparison of ionospheric dynamo currents and magnetic perturbations modeled by the TIEGCM with CM3e model results, EGS - AGU - EUG Joint Assembly, Nice, France, 6-11 April 2003, EAE03A-04686, 2003.

Olsen, N., Lühr, H., Sabaka, T., Mandea, M., Rother, M., TøffnerClausen, L., and Choi, S.: CHAOS - A Model of Earth's Magnetic Field derived from CHAMP, Ørsted, and SAC-C magnetic satellite data, Geophys. J. Int., 166, 67-75, doi:10.1111/j.1365246X.2006.02959.x, 2006.

Reigber, Ch., Lühr, H., and Schwintzer, P.: CHAMP Mission Status, Adv. Space Res., 30(2), 129-134, 2002.

Richmond, A. D.: The computation of magnetic effects of fieldaligned magnetospheric currents, J. Atmos. Terr. Phys., 36, 245252, 1974.

Richmond, A. D.: Modeling the Ionosphere Wind Dynamo: A Review, PAGEOPH, 131(3), 413-435, 1989.

Richmond, A. D., Ridley, E. C., and Roble, R. G.: A thermosphere ionosphere general circulation model with coupled electrodynamics, Geophys. Res. Lett., 19, 601-604, 1992.

Richmond, A. D.: Ionospheric electrodynamics using magnetic apex coordinates, J. Geomagn. Geoelectr., 47, 191-212, 1995.

Richmond, A. D.: Modeling the geomagnetic perturbations produced by ionospheric currents, above and below the ionosphere, J. Geodyn., 33, 143-156, 2002.

Schachtschneider, R., Balasis, G., Rother, M., and Mandea, M.: Wavelet-based selection of satellite data for geomagnetic core field modeling, in Proceedings of the First Swarm International Science Meeting, Nantes, France, 3-5 May 2006, ESA WPP261, July 2006.

Tyler, R. H., Maus, S., and Lühr, H.: Satellite observations of magnetic fields due to ocean tidal flow, Science, 299, 239-241, 2003. 